\title{
Alternatives For Incorporating Opportunity Cost Of Time In Recreation Demand Models
}

Chris Azevedo, University of Central Missouri, USA

\begin{abstract}
The importance of accounting for a respondent's travel time in recreation demand models is well established. In practice, most analysts use a fixed fraction of the respondent's wage rate to value travel time. However, other approaches have been suggested in the literature. In this paper revealed and stated preference data on Iowa wetland usage is used to explore various specifications of travel time. It is shown that the choice of a particular specification has a direct impact on welfare estimates as well as the consistency between revealed and stated preference data.
\end{abstract}

Keywords: Recreation Demand; Opportunity Cost of Time; Non-Market Valuation

\section{INTRODUCTION}

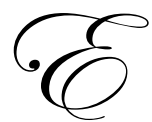

conomists have long recognized the important role played by the opportunity cost of time in recreation demand models. This importance derives primarily from the time-intensive nature of goods such as outdoor recreation. The time spent engaging in recreation is an important component of the travel cost or site "price", the correct specification of which, is instrumental in calculating accurate welfare measures. The important role of the opportunity cost of time is highlighted by Bishop and Heberlein (1979), who find that valuing travel time at half the wage rate, as opposed to not including it at all, results in a fourfold difference in consumer surplus estimates for their application.

Despite recognizing its importance, no consensus has been reached as to the appropriate method of dealing with travel time. The problem was first identified by Cesario (1976), where he used a fixed ratio of the wage rate to value time. McConnell and Strand (1981) generalized this approach by estimating the implicit value of time directly from the data. Bockstael et al. (1987) recognized that the wage rate might not provide information about the respondent's opportunity cost of time in cases where the respondent faces a fixed work week. Other researchers took a slightly different approach by developing hedonic wage models (Smith et al. (1983), Feather and Shaw (1999)).

Larson and Shaikh (2004) develop an innovative model in which an endogenous value of time function is estimated jointly with the recreation demand problem. Time and budget constraints are incorporated into the consumer's problem in a utility-consistent manner and the properties of the two-constraint problem are explored. Additionally, their parametric specification of the endogenous value of time function nests the estimation approaches described above. This is useful in that it allows for hypothesis tests of the appropriateness of the various models that have been developed in the literature.

Despite these advances in the literature, most researchers choose to bypass the issue by using a fixed fraction of the wage rate, usually one third, at which to value time. This pragmatic approach seems to stem from Cesario's (1976, p. 37) suggestion that 'it may be tentatively concluded that the value of time with respect to nonwork travel is between one fourth and one half of the wage rate'. 
Coinciding with the developments in the opportunity cost of time research mentioned above have been developments in the literature concerning the relationship between revealed preference (RP) data and stated preference (SP) data. Prior to Cameron (1982), most researchers viewed RP and SP as competing methodologies for valuation. Cameron's insight was that the two types of data could be combined in order to take advantage of the strengths of each methodology. This approach has been used with discrete models (Adamowicz et al. (1994), Loomis (1997)), continuous models (Dickie et al. (1987)), as well as models that have both a discrete and continuous portion (Larson (1990)).

In this paper I examine several options for parametrically specifying the opportunity cost of travel time, similar to the approach taken by Larson and Shaikh. Further, each option will be examined using the three methodologies for valuation discussed above: revealed preference data, stated preference data, and a model that links the two sources of data. These methodologies can be thought of as three different "laboratories" in which I can investigate the consequences of alternative treatments of time costs.

The next section will be used to describe the methods of incorporating time into the recreation demand model. The three laboratories will then be discussed as well as the forms of data used to estimate each model. Parameter estimates will be presented and the implications of the results will be explored.

\section{METHODS OF INCORPORATING TIME INTO THE RECREATION DEMAND MODEL}

In this paper I'll examine four methods of incorporating time into the recreation demand model: picking a fixed fraction of the wage rate, estimating the fraction of the wage rate without accounting for the employment status of the respondent, estimating the fraction of the wage rate while explicitly accounting for the employment status of the respondent, and the more general approach developed by Bockstael et al. (1987).

The first method to be examined is the use of a fixed fraction of the wage rate. The majority of past authors have chosen to model the opportunity cost of travel time as some fixed fraction of the full wage rate. Cesario (1976), in a survey of empirical evidence concerning urban commuters, concluded that the opportunity cost of travel time was between one-fourth and one-half of the wage rate. Based on this evidence he concluded that it would be reasonable to value travel time at one-third the wage rate. Although clearly ad hoc, this method has the advantage of simplicity.

The second method I will examine is direct estimation of the marginal opportunity cost of travel time. McConnell and Strand (1981) develop a model that explicitly estimates the fraction of the full wage rate at which time is valued by adding that fraction as a parameter to be estimated. This approach is more appealing than the assertion of a fixed fraction of the wage rate, but has not enjoyed common usage due to difficulties with collinearity. Although I did not find these difficulties in my applications, I briefly discuss this issue later.

The third method is akin to the McConnell and Strand (1981) approach, but estimates a separate fraction of the wage rate for respondents who can alter their work hours at the margin and for respondents who must work a fixed number of hours. This allows more flexibility for the data to yield information on the opportunity cost of time.

The final method I will consider was developed in Bockstael, Strand, and Hanemann (1987). Bockstael et al. develop a model that is similar to the McConnell and Strand (1981) model, except they take a closer look at the structure of the time constraint. They point out that the nature of an individual's labor supply decision determines whether their wage rate yields information about the marginal value of their time. It may not be possible for a respondent to optimally adjust the number of hours worked. If this is the case, they will be found at a corner solution where they choose either to not work, or to work a job with a fixed number of hours. The respondent may choose to work a part-time job with a flexible number of hours in addition to their job with fixed hours, or they may choose not to work at all.

In general, these models progress from ad hoc to more rigorous treatments of the opportunity cost of travel time. Whether the more rigorous treatments yield vastly different empirical results than the simpler methods is the focus of this paper. 


\section{Empirical Models}

Three separate laboratories will be used to estimate the models. Each model will be estimated using revealed preference data alone, stated preference data alone, and both revealed and stated preference data in a linked model. In this Section I will describe the RP model, the SP model, and the model that links both RP and SP data.

\section{Laboratory 1: Revealed Preference Data}

The demand model describing the RP data assumes an individual allocates income between a composite commodity $(z)$ and a recreation good $(q)$. The ordinary demand (Marshallian) associated with the recreation good can be written simply as

$q_{i}^{R}=f^{R}\left(p_{i}^{R}, y_{i} ; \beta^{R}\right)+\varepsilon_{i}^{R}$,

where $q_{i}^{R}$ is the quantity consumed by individual $i, p_{i}^{R}$ denotes the associated price, $y_{i}$ is the individual's income, and $\beta^{R}$ is a vector of unknown parameters. The additive stochastic term is assumed to follow a normal distribution, with $\varepsilon_{i}^{R}$ distributed $N\left(0, \sigma_{R}^{2}\right)$. Since LHS censoring is present in my data (as in many recreation demand applications), standard econometric estimators are used to obtain consistent estimates of the parameters of this function accounting for censoring. Specifically, the likelihood function is written

$$
L L^{R}=\sum_{i=1}^{n}\left(D_{i}^{R} \ln \left\{\sigma_{R}^{-1} \phi\left[\frac{q_{i}^{R}-f^{R}\left(p_{i}^{R}, y_{i} ; \beta^{R}\right)}{\sigma_{R}}\right]\right\}+\left(1-D_{i}^{R}\right) \ln \left\{\Phi\left[\frac{-f^{R}\left(p_{i}^{R}, y_{i} ; \beta^{R}\right)}{\sigma_{R}}\right]\right\}\right)
$$

where $\Phi$ and $\phi$ are the standard normal cdf and pdf, respectively, and $D_{i}^{R}=1$ if $q_{i}^{R}>0 ;=0$ otherwise.

\section{Laboratory 2: Stated Preference Data}

Now suppose that in the process of gathering RP data, the survey respondents are asked: "How many recreation trips would you have taken to this site if the cost per trip increased by $\$ B$ ?" The response to this question represents a form of SP data. I will have both quantity $\left(q_{i}^{S}\right)$ and price $\left(p_{i}^{S}\right)$ information for each individual. If, as in the case of the RP data, I assume that the survey responses are driven by an underlying set of preferences, the stated demands flow from demand equations of the form $q_{i}^{S}=f^{S}\left(p_{i}^{S}, y_{i} ; \beta^{S}\right)+\varepsilon_{i}^{S}$, where $p_{i}^{S}=p_{i}^{R}+B_{i}$.

Having constructed the log-likelihood function for the RP data, it is quite straightforward to construct it for the SP data since they are of identical form. Thus, the log-likelihood function in Equation (2) will also describe the SP data, requiring only that $R$ be replaced with $S$ everywhere.

\section{Laboratory 3: Linking Revealed and Stated Preference Data}

The past several years have seen a change in the research agenda of environmental valuation. Rather than treating RP and SP as competing valuation techniques, analysts have begun to view them as complementary, where the strengths of each approach can be used to provide more precise and possibly more accurate benefit estimates. The impetus for this change was a paper by Cameron (1992) where she combined information on the number of fishing trips in Southern Texas with responses to an SP question regarding the angler's willingness-to-pay for annual angling. She notes that the same set of preferences that generate the RP data ought also to generate the SP data. Thus, both sources of data yield information on a common set of parameters. There are now numerous examples of authors using both RP and SP data to jointly estimate the parameters of a preference function (McConnell, et al. 1999, Adamowicz et al. 1994, Larson 1990). 
If the RP and SP data are to be linked in joint estimation of preferences, efficiency would dictate that I take into account the likely correlation between the RP and SP responses. The log likelihood function is given by ${ }^{1}$

$$
\begin{aligned}
L L= & \sum_{i=1}^{n}\left\{D_{i}^{R}\left[\ln \phi\left(\frac{q_{i}^{R}-f_{i}^{R}}{\sigma_{R}}\right)-\ln \left(\sigma_{R}\right)\right]+D_{i}^{R} D_{i}^{S}\left[\ln \phi\left(\frac{\left(q_{i}^{S}-f_{i}^{S}\right)-\theta^{S}\left(q_{i}^{R}-f_{i}^{R}\right)}{\sigma_{S} \sqrt{1-\rho^{2}}}\right)-\ln \left(\sigma_{S} \sqrt{1-\rho^{2}}\right)\right]\right. \\
& \left.+D_{i}^{R}\left(1-D_{i}^{S}\right)\left[\ln \Phi\left(\frac{-f_{i}^{S}-\theta^{S}\left(q_{i}^{R}-f_{i}^{R}\right)}{\sigma_{S} \sqrt{1-\rho^{2}}}\right)\right]+\left(1-D_{i}^{R}\right)\left(1-D_{i}^{S}\right) \ln \int_{-\infty}^{\frac{-f_{i}^{R}}{\sigma_{R}}-f_{i}^{S}} \frac{\int_{-\infty}^{\sigma_{S}}}{\sigma_{2}} \phi_{2}\left(\eta_{1}, \eta_{2} ; \rho\right) d \eta_{1} d \eta_{2}\right\}
\end{aligned}
$$

where $\rho \equiv \operatorname{Corr}\left(\varepsilon_{i}^{R}, \varepsilon_{i}^{S}\right), \theta \equiv \rho \sigma_{S} / \sigma_{R}, f_{i}^{k}=f^{k}\left(p_{i}^{k}, y_{i}^{k} ; \beta^{k}\right)(k=R, S)$, and $\phi_{2}(\cdot, ; \rho)$ denotes the standard normal bivariate pdf. This model can be used to test a variety of hypotheses concerning the consistency of the RP and SP data. All of the coefficients entering the SP portion of the likelihood can be constrained to be the same as those in the RP portion, they can all be allowed to differ, or some subset can be constrained to be equal across the data sources. The parameter estimates reported in this paper will be for a linking model that restricts all RP and SP parameters to be equal.

\section{Parametric Specifications of Time}

The four models I am considering differ according to the specification of the variables related to the time cost in the demand function.

\section{Model 1: Fixed Marginal Opportunity Cost of Time}

In the first case, a fixed marginal opportunity cost of time is used. The trip demand function takes the form

$$
q_{i}^{j}=\alpha^{j}+\beta_{p}^{j} p_{i}^{j}+\beta_{y}^{j} y_{i}+\varepsilon_{i}^{j}
$$

where $\varepsilon_{i}^{R}$ is distributed $N\left(0, \sigma_{R}^{2}\right)$ and $j=R, S$. The price term takes the form $p_{i}^{j}=C_{i}+(1 / 3) w_{i} T_{i}$, where $C_{i}$ denotes out-of-pocket travel expense, $w_{i}$ denotes the wage rate, and $T_{i}$ is round-trip travel time. The marginal opportunity cost of time is assumed to be one-third of the wage rate for all recreators, regardless of their employment status or ability to work additional hours.

Model 2: Estimating a Single Marginal Opportunity Cost of Time

The second model allows the marginal opportunity cost of travel time to be estimated as a parameter in the model. In this case the price specification takes the form

$p_{i}^{j}=C_{i}+\lambda^{j} w_{i} T_{i}$

where $\lambda^{j}$ is the proportion of the wage at which travel time is valued. A single $\lambda^{j}$ is estimated for all respondents, again imposing that the rate is fixed across all recreators.

\footnotetext{
${ }^{1}$ The derivation of this log-likelihood function is available from the author upon request.
} 
Model 3: Accounting for Employment Status, First Approach

The third model estimates a different $\lambda^{j}$ for respondents who can optimally adjust their work hours at the margin than for respondents who must work at a job with a fixed number of hours. In this case the price specification takes the form

$p_{i}^{j}=C_{i}+I_{i} \lambda_{a}^{j} w_{i} T_{i}+\left(1-I_{i}\right) \lambda_{f}^{j} w_{i} T$,

where $I_{i}$ is an indicator variable that takes a value of unity if respondent $i$ can optimally adjust their work hours and a value of zero if they must work a fixed number of hours, $\lambda_{a}^{j}$ is the marginal opportunity cost of time for respondents who can adjust their work hours, while $\lambda_{f}^{j}$ is the marginal opportunity cost of time for respondents who must work a fixed number of hours.

This approach allows for some flexibility, but is still a rather ad hoc method of accounting for the employment status of the respondent.

\section{Model 4: Accounting for Employment Status, Bockstael et al. Model}

The final model I will examine is Bockstael, Strand, and Hanemann (1987). The essence of the Bockstael et al. model is that respondents face both a time and income constraint. If the respondent can freely substitute time for money, the two constraints can be collapsed. However, if the respondent cannot freely substitute time for money the constraints cannot be collapsed. This implies that the structure of the demand function will be different for the two cases.

I will estimate the linear model developed in the Bockstael et al. paper. The trip demand function for respondents who can optimally adjust their work hours takes the form

$q_{i}^{j}=\alpha^{j}+\gamma_{1}^{j}\left(y_{i}+w_{i} \bar{T}_{i}\right)+\beta^{\prime j} \gamma_{2}^{j}\left(C_{i}+w_{i} T_{i}\right)+\varepsilon_{i}^{j}$,

where $\bar{T}_{i}$ represents discretionary time (time spent not working) and $\beta^{j j}=\beta^{j} /\left(\gamma_{1}^{j}+\gamma_{2}^{j}\right)$. The trip demand function for respondents who cannot optimally adjust their work hours takes the form

$q_{i}^{j}=\alpha^{j}+\gamma_{1}^{j} y_{i}+\gamma_{2}^{j} \bar{T}_{i}+\beta^{\prime j} \gamma_{1}^{j} C_{i}+\beta^{\prime j} \gamma_{2}^{j} T_{i}+\varepsilon_{i}^{j}$.

The important distinction between this model and the previous three is that the wage does not enter the demand function of respondents who cannot optimally adjust their work hours.

\section{AN APPLICATION TO WETLANDS IN IOWA}

These models will be applied using data from a 1997 survey of Iowa residents concerning their use of Iowa wetlands. Of the 6,000 surveys sent, 594 were returned by the post office as undeliverable. There was a 59 percent response rate (with 3,143 surveys returned). The survey instrument elicited travel cost information, contingent behavior information in both continuous and discrete form, as well as socioeconomic information (e.g., gender, age, and income). A complete discussion of the wetland data set can be found in Azevedo (2000).

The state of Iowa was divided into fifteen zones, shown in Figure 1. These zones each contained between 3 and 12 counties, and were designed to encompass similar types of wetlands. For analysis, the zones were further grouped into "megazones" with each megazone containing three zones. Zones 1, 2, and 3 comprise the 1,2,3 megazone, zones 4,5 , and 8 comprise the 4,5,8 megazone, zones 6,7 , and 12 comprise the 6,7,12 megazone, zones 9,10 , and 11 comprise the 9,10,11 megazone, and zones 13,14 , and 15 comprise the 13,14,15 megazone. For this analysis only the data from zones 4, 5, and 8 (4,5,8 megazone) were used. 


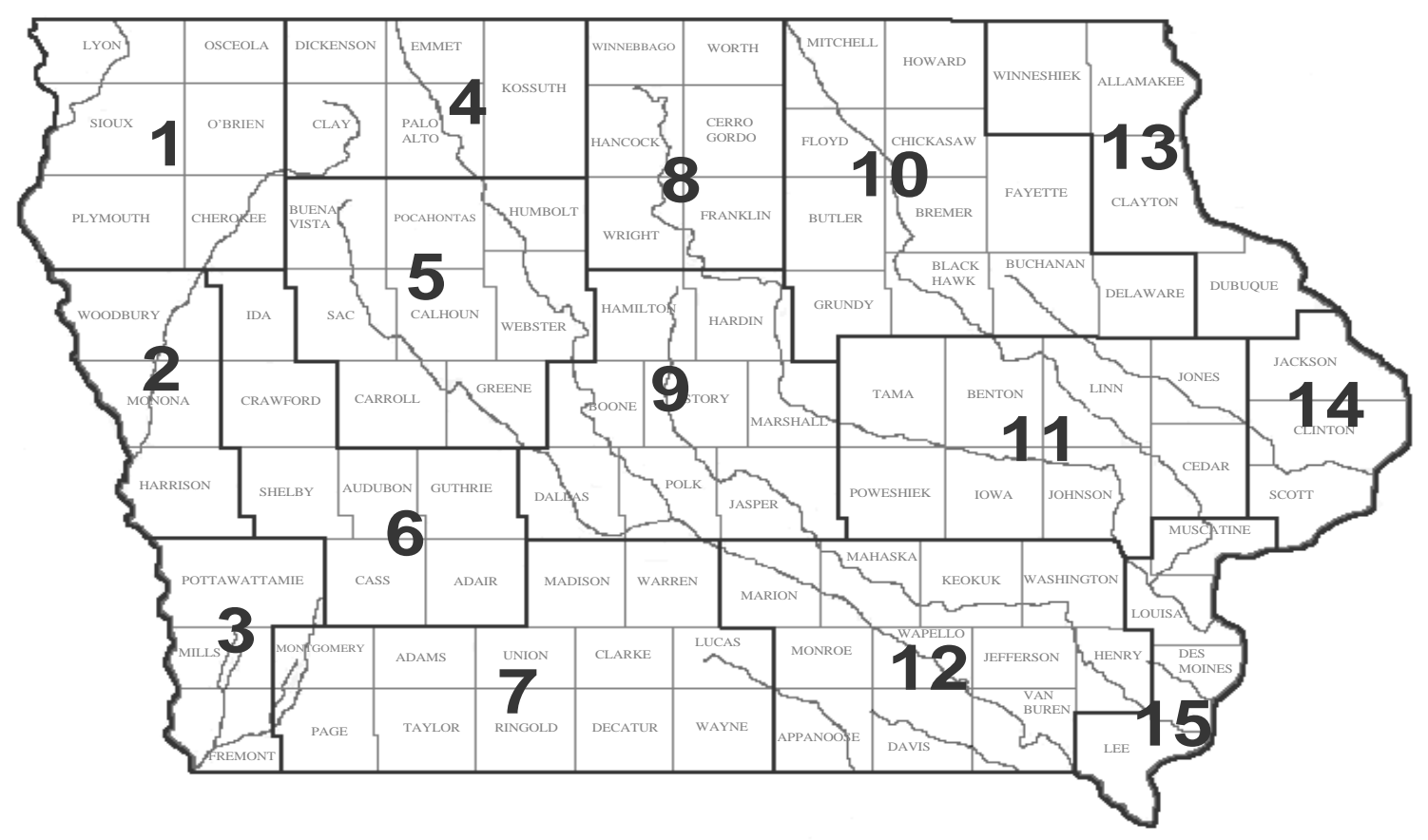

Figure 1: Iowa Wetland Zones 


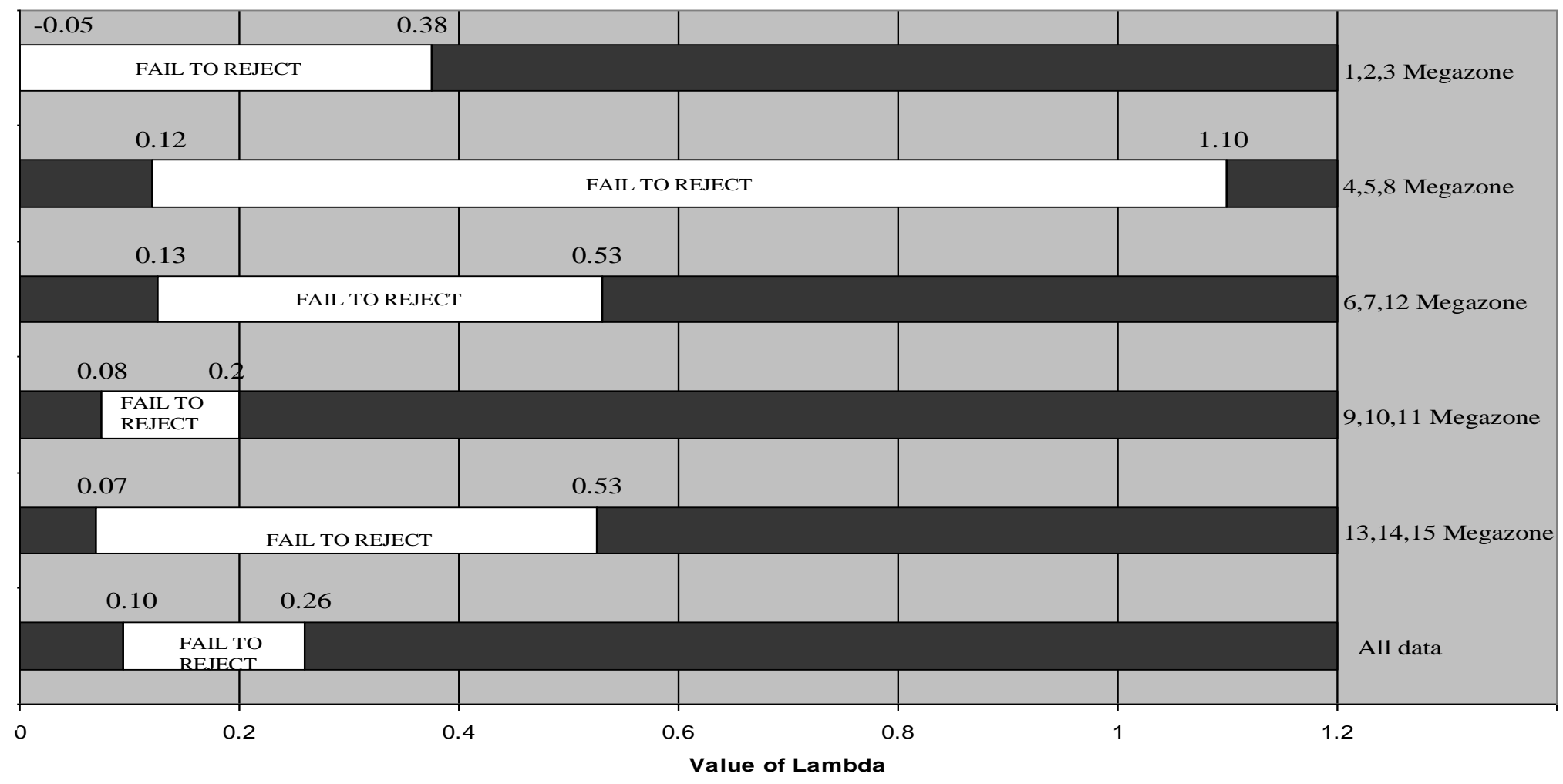

Figure 2: Testing general consistency with fixed lambda 
One section of the survey asked respondents to indicate the number of trips they had taken to each of the fifteen zones over the past year. This provided the RP data for my analysis. The respondents were then asked to consider a \$B increase in the total cost per trip of each of the trips they had taken in 1997, and asked the following SP question concerning the trips they made to zones near their residence (X, Y, and Z for illustration): "With this additional cost of $\$ \mathrm{~B}$ per trip of visiting zones $\mathrm{X}, \mathrm{Y}$, and $\mathrm{Z}$, would this affect the number of trips you made to any of the 15 zones?" They were then asked to elaborate on how many fewer trips they would have taken to each of zones $\mathrm{X}, \mathrm{Y}$, and $\mathrm{Z}$. The bid values $(\$ \mathrm{~B})$ were varied randomly across the sample, ranging from $\$ 5$ to $\$ 50$. This provided the data for the SP model.

The surveys provided direct information on the trip quantities. The next step was to calculate the out-ofpocket cost of travel as well as the travel time associated with visiting each zone. I used the software package PC Miler, designed for use in the transportation and logistics industry, to establish both travel distance $\left(d_{i}^{z}\right)$ and time $\left(T_{i}^{z}\right)$ for each household from their residence to the center of each wetland zone. The price of visiting a given wetland zone $z$ was then constructed as $C_{i}^{z}=0.22 d_{i}^{z}$.

Summary statistics for the data used in this analysis are provided in Table 1. The average out-of-pocket travel cost, $C_{i}$, was $\$ 22.57$. Average round trip travel time, $T_{i}$, was 1.31 hours, with an average number of trips take within the 4,5,8 megazone of 8.28. After the price increase, the average out-of-pocket travel cost was $\$ 47.76$, with an average quantity of trips at the new price of 2.72 .

Table 1: Iowa wetlands data set, summary statistics for $4,5,8$ megazone

\begin{tabular}{|l|c|}
\hline Number of respondents & 274 \\
\hline Average out-of-pocket travel cost & $\$ 22.57$ \\
\hline Average round trip travel time & 1.31 hours \\
\hline Average quantity of trips taken to this megazone & $\$ 4.28$ \\
\hline Average out-of-pocket travel cost with price increase & 2.72 \\
\hline Average quantity of trips after price increase & $\$ 37,4995$ \\
\hline Median income & \\
\hline
\end{tabular}

\section{PARAMETER ESTIMATES}

Table 2 shows parameter estimates for each of the four models. The most striking aspect of these results is the difference in the estimates of $\lambda$ between the RP and SP data. Model 2 estimates a revealed preference $\lambda$ of 0.06 (not significantly different from zero), while the stated preference $\lambda$ is 0.43 . Model 3 also estimates revealed preference $\lambda$ 's very near zero with stated preference $\lambda$ 's significantly larger. This implies that the practice of using a fixed $\lambda$, often chosen at one-third, would likely be more problematic with the RP data.

Another interesting result is that the estimates of $\lambda_{f}$ and $\lambda_{a}$ (Model 3) are very similar. In the RP laboratory, the estimate for $\lambda_{f}$ is 0.002 while the estimate for $\lambda_{a}$ is 0.00 . In both the SP and RP-SP laboratories the estimates for $\lambda_{f}$ and $\lambda_{a}$ are slightly different ( 0.48 vs. 0.41 in the SP case and 0.48 vs. 0.42 in the RP-SP case) but still very close. This indicates that with respect to the marginal opportunity cost of time, for this demand specification, there does not appear to be much difference between respondents who can adjust their work hours and those who cannot.

All models exhibit a high degree of correlation between the RP and SP data sets, as shown by the estimates of $\rho$ in Laboratory 3. Parameter estimates are 0.70 for Model 1, 0.72 for Model 2, 0.72 for Model 3, and 0.64 for Model 4. 


\section{IMPLICATIONS: WELFARE MEASURES AND RP-SP CONSISTENCY}

Table 2 also shows that the choice of model can have a significant effect on the consumer surplus measure. Within the RP Laboratory, fixing the marginal opportunity cost of time at one-third resulted in a consumer surplus of 185.12, significantly larger than the consumer surplus measures of the other three models $(82.80$ for Model 2, 93.11 for Model 3, and Model 4 estimates of 148.17 for respondents with flexible work hours and 118.50 for respondents with fixed work hours).

Table 2: Parameter Estimates (t-statistic in parenthesis)

\begin{tabular}{|c|c|c|c|}
\hline & $\begin{array}{c}\text { Laboratory } 1 \\
\text { RP } \\
\end{array}$ & $\begin{array}{c}\text { Laboratory } 2 \\
\text { SP } \\
\end{array}$ & $\begin{array}{c}\text { Laboratory } 3 \\
\text { RP-SP }\end{array}$ \\
\hline \multicolumn{4}{|c|}{ Model 1: Fixed $\lambda=1 / 3$ : } \\
\hline$\alpha$ & $15.69(7.20)^{* *}$ & $10.57(2.66)^{* *}$ & $14.79(7.64) * *$ \\
\hline$\beta$ & $-0.52(-7.68)^{* *}$ & $-0.45(-5.99)^{* *}$ & $-0.50(-15.40)^{* * *}$ \\
\hline$\gamma$ & $0.18(4.81)^{* *}$ & $0.18(3.20)^{* *}$ & $0.18(5.01)^{* *}$ \\
\hline$\sigma$ & $13.79(18.09)^{* *}$ & $15.26(10.97)^{* *}$ & $14.12(18.97)^{* *}$ \\
\hline$\rho$ & -- & -- & $0.70(16.33)^{* *}$ \\
\hline $\mathrm{CS}$ & 185.12 & 216.53 & 188.03 \\
\hline RP-SP consistency & & & Fail to reject \\
\hline \multicolumn{4}{|l|}{ Model 2: Estimating $\lambda$} \\
\hline$\alpha$ & $27.11(8.77)^{* *}$ & $9.77(2.31)^{*}$ & $14.52(7.48)^{* *}$ \\
\hline$\beta$ & $-1.15(-8.37)^{* *}$ & $-0.42(-4.64)^{* *}$ & $-0.47(-11.34)^{* *}$ \\
\hline$\lambda$ & $-0.06(-1.59)$ & $0.43(2.39)^{*}$ & $0.44(3.93)^{* *}$ \\
\hline$\gamma$ & $-0.02(-0.32)$ & $0.20(2.92)^{* *}$ & $0.21(4.29)^{* *}$ \\
\hline$\sigma$ & $13.26(18.30)^{* * *}$ & $15.31(11.25)^{* * *}$ & $14.34(18.40)^{* * *}$ \\
\hline$\rho$ & -- & -- & $0.72(16.28)^{* *}$ \\
\hline $\mathrm{CS}$ & 82.80 & 239.85 & 197.39 \\
\hline RP-SP consistency & & & Reject \\
\hline \multicolumn{4}{|c|}{ Model 3: Different $\lambda$ 's } \\
\hline$\alpha$ & $25.04(8.90)^{* *}$ & $9.77(2.41)^{*}$ & $14.33(7.03)^{* *}$ \\
\hline$\beta$ & $-1.03(-8.61)^{* *}$ & $-0.41(-4.70)^{* *}$ & $-0.47(-11.69)^{* *}$ \\
\hline$\lambda_{f}$ & $0.002\{8.34\}^{* * 1}$ & $0.48\{0.90\}^{1}$ & $0.48\{0.89\}^{1}$ \\
\hline$\lambda_{a}$ & $0.000(0.08)$ & $0.41(2.36)$ & $0.42(3.57)^{* *}$ \\
\hline$\gamma$ & $0.04(1.10)$ & $0.20(3.10)$ & $0.22(3.93)^{* *}$ \\
\hline$\sigma$ & $13.33(18.30)$ & $15.27(10.46)$ & $14.31(17.74)^{* *}$ \\
\hline$\rho$ & -- & -- & $0.72(15.25)^{* *}$ \\
\hline $\mathrm{CS}$ & 93.11 & 242.68 & 199.66 \\
\hline RP-SP consistency & & & Reject \\
\hline \multicolumn{4}{|c|}{ Model 4: Bockstael et al. } \\
\hline$\alpha$ & $11.89(5.76)^{* *}$ & $8.95(133.91)^{* * *}$ & $10.82(4.82)^{* * *}$ \\
\hline$\gamma_{1}$ & $0.07(4.46)^{* *}$ & $0.04(11.71)^{* *}$ & $0.06(4.50)^{* *}$ \\
\hline$\gamma_{2}$ & $0.32(0.96)$ & $1.90(25.10)^{* *}$ & $0.57(2.48)^{*}$ \\
\hline$\beta^{\prime}$ & $-7.18(-6.11) * *$ & $-6.82(-101.21)^{* *}$ & $-7.17(-4.86)^{* *}$ \\
\hline$\sigma$ & $12.60(15.24)^{* * *}$ & $10.65(157.04)^{* * *}$ & $12.56(16.01)^{* *}$ \\
\hline$\rho$ & -- & -- & $0.64(10.31)^{* *}$ \\
\hline CS: flexible & 148.17 & 252.95 & 171.24 \\
\hline CS: fixed & 118.50 & 202.29 & 136.95 \\
\hline RP-SP consistency & & & Fail to reject \\
\hline
\end{tabular}

** Denotes significance at the $99 \%$ confidence level, $*$ Denotes significance at the $95 \%$ confidence level

1 t-statistic for a hypothesis test of significant difference between $\lambda_{f}$ and $\lambda_{a}$ 
Within Laboratory 2, consumer surplus ranges from a low of 202.29 (Model 4, CS: fixed) to 242.68 (Model 3). Within Laboratory 3 , there is very little difference between the consumer surplus estimates of Models 1 through 3 (188.03, 197.39, and 199.66 respectively). However, Model 4 estimates are slightly lower (171.24 and 136.95). In general, RP data alone (Laboratory 1) produces lower consumer surplus estimates than SP data alone (Laboratory 2), with RP-SP (Laboratory 3) falling between the two.

The modeling choice can also have a significant effect on the hypothesis test of consistency between RP and SP data. The linked RP-SP model can be used to test the hypothesis of parameter equality between the RP and SP data sets. ${ }^{2}$ With Models 2 and 3, the null hypothesis of parameter equality between the revealed and stated preference data sets was rejected. However, with Models 1 and 4, the null hypothesis of parameter equality was not rejected.

To further investigate the effect of choosing a fixed $\lambda$, a search procedure was conducted that tested consistency between revealed and stated preference data using a different value of $\lambda$ for each test. Figure 2 shows the result of this search procedure for each megazone as well as for the overall data set.

For each group of data there exists a range of values of $\lambda$ that will result in a failure to reject the null hypothesis of consistency between the revealed and stated preference data. The "fail to reject" region for the 4,5,8 data (the data used in this analysis) includes values of $\lambda$ between 0.12 and 1.10. Four out of the five megazones (1,2,3 megazone, 4,5,8 megazone, 6,7,12 megazone, and the 13,14,15 megazone) include the value of one-third in the consistency region. The only megazone for which a value of $\lambda$ equal to one-third results in a rejection of consistency is the $9,10,11$ megazone.

This is a very important result. Testing for consistency between revealed and stated preference data is often a primary goal of papers that link both forms of data. As these results show, the choice of model can have a significant impact on the outcome of hypothesis tests of consistency between revealed and stated preference data. When estimating the model with a fixed $\lambda$, the consistency results depend on whether the value of $\lambda$ chosen falls into the range of "consistent $\lambda$ 's" for that data set. However, if the opportunity cost of travel time is added as a parameter to be estimated, all tests result in a rejection of the null hypothesis of consistency. Consistency tests for Model 4 resulted in a failure to reject the hypothesis of consistency.

\section{FINAL COMMENTS}

In this paper I have examined the modeling of travel time in the recreation demand model. Four separate models were each considered in one of three laboratories. It was shown that the way the opportunity cost of time is modeled in the recreation demand model can have a significant impact both on the estimates of consumer surplus and the hypothesis tests of consistency between revealed and stated preference data.

\section{AUTHOR INFORMATION}

Chris Azevedo, Dept. of Economics and Finance, University of Central Missouri. Dr. Azevedo earned his Ph.D. at Iowa State University. He is currently an associate professor of economics at the University of Central Missouri in Warrensburg, Mo. E-mail: cazevedo@ucmo.edu

\section{REFERENCES}

1. Adamowicz, W., Louviere, J., and Williams, M. "Combining Revealed and Stated Preference Methods for Valuing Environmental Amenities.” Journal of Environmental Economics and Management 10(1994): 271292.

\footnotetext{
${ }^{2}$ Restricted (RP and SP parameters equal) and unrestricted models were estimated, and a likelihood ratio statistic was used to test the null hypothesis of parameter equality between the RP and SP data sets.
} 
2. Azevedo, C. D. Linking Revealed and Stated Preference Data in Recreation Demand Modeling, Ph.D. Dissertation, Iowa State University (2000).

3. Bishop, R. and Heberlein T. "Travel Cost and Hypothetical Valuation of Outdoor Recreation: Comparisons with an Artificial Market.” Dep. Agr. Working Paper, University of Wisconsin (1979).

4. Bockstael, Strand, and Hanemann "Time and the Recreation Demand Model" American Journal of Agricultural Economics 69(1987): 293-302.

5. Cameron, T. "Combining Contingent Valuation and Travel cost Data for the Valuation of Nonmarket Goods." Land Economics 68(1992): 302-317.

6. Cesario, F. J., "Value of Time in Recreation Benefit Studies.” Land Economics 52(1976):32-41.

7. Feather, P. and Shaw W. D. "Estimating the Cost of Leisure Time for Recreation Demand Models" Journal of Environmental Economics and Management 38(1999): 49-65.

8. Larson, D. "Testing Consistency of Direct and Indirect Methods for Valuing Nonmarket Goods." Working Paper 1990

9. Larson, D. and Sabina L. Shaikh, "Recreation Demand Choices and Revealed Values of Leisure Time," Economic Inquiry, 42(2004): 264-278.

10. McConnell, K., and Strand, I. "Measuring the Cost of Time in Recreation Demand Analysis: An Application to Sportfishing." American Journal of Agricultural Economics 63(1981): 153-156.

11. McConnell, K., Weninger, Q., and Strand, I. "Joint Estimation of Contingent Valuation and Truncated Recreational Demands." Valuing Recreation and the Environment (1999).Smith, Desvousges, and McGivney

12. Smith, V. K., Desvousges, W. H., and McGivney, M. P. "The opportunity cost of travel time in recreational demand models," Land Economics. 59(3): 259-277, (1983). 


\section{$\underline{\text { NOTES }}$}

